\title{
ON THE CYCLIC CONNECTIVITY THEOREM*
}

\author{
BY G. T. WHYBURN
}

1. Introduction. We shall call the following theorem the cyclic connectivity theorem.

Every two points of a locally connected continuum having no cut point lie together on a simple closed curve in that continuum.

The demonstration for this theorem originally given by the present author $\dagger$ for the case of plane continua and in particular the demonstration given later by Ayres $\neq$ for the theorem in general space are undeniably quite complicated. Indeed, the complexity of the proof of this theorem constituted a strong incentive to the author to seek and find $\S$ a new treatment of the cyclic element theory which not only avoids using this theorem as principal point of departure as does the original one $\|$ but also has validity in all connected, locally connected, metric, and separable spaces, and thus in spaces in which the proposition in question obviously does not hold. The same complexity was the prevailing influence motivating a development by Kuratowski and the author 1 of most of the cyclic element theory for compact locally connected continua in a simple and direct way independent of the cyclic connectivity theorem, based on a definition of cyclic element suggested by R. L. Moore.**

Thus it is seen that although this proposition has been almost successfully avoided in so far as the cyclic element theory is

\footnotetext{
* Presented to the Society, February 28, 1931.

$\dagger$ See Proceedings of the National Academy of Sciences, vol. 13 (1927), pp. 31-38. 594.

$\ddagger$ W. L. Ayres, American Journal of Mathematics, vol. 51 (1929), pp. 577-

§ See Transactions of this Society, vol. 32 (1930), pp. 926-943.

\| See American Journal of Mathematics, vol. 50 (1928), pp. 167-194.

१ C. Kuratowski et G. T. Whyburn, Sur les éléments cycliques et leurs applications, Fundamenta Mathematicae, vol. 16 (1930), pp. 305-331. The authors of this article describe the proof of the cyclic connectivity theorem as being "fort compliquée."

** R. L. Moore, Monatshefte für Mathematik und Physik, vol. 36 (1929), pp. 81-88.
} 
concerned, it has not been proved in the direct and simple manner which is characteristic of demonstrations for the majority of theorems concerning cyclic elements. The present paper offers as its principal contribution just such a demonstration for this theorem, based on a small amount of the cyclic element theory which, for the sake of completeness, is appended at the end of the paper, together with a few fundamental and long established properties of locally connected continua. The cyclic connectivity theorem thus finds its proper place in the subject as an important consequence of the cyclic-element decomposition of locally connected continua and an important complement to the cyclic element theory.

2. The Proof. Let $M$ designate any locally connected, locally compact, separable and metric continuum, which we shall consider as a space, and let $C$ designate any such space which has no cut point.

Lemma 1. If $A$ and $B$ are non-degenerate, ${ }^{*}$ closed and mutually exclusive subsets of $C$, there exist two mutually exclusive arcs in $C$ joining $A$ and $B$.

There exists an arc $a b$ in $C$ where $a b \cdot A=a, a b \cdot B=b$; and if $p$ is a point of $A-a$, clearly there exist points $x$ which can be joined in $C$ to $p$ by an arc $p x$ containing no point of $a b$. Thus there exist points $x$ such that mutually exclusive arcs $a b$ and $p x$ exist in $C$ so that

$$
a b \cdot A \supset a, a b \cdot B \supset b \text {, and } p x \cdot A \supset p .
$$

Let $S$ denote the set of all such points $x$. I shall show that $S=C$. Suppose this is not so. Then since $C$ is connected and clearly $S$ is open in $C$, it follows that at least one point $y$ of $C-S$ is a limit point of $S$. There exists an arc $a^{\prime} b^{\prime}$ in $C-y$ such that $a^{\prime} b^{\prime} \cdot A=a^{\prime}$ and $a^{\prime} b^{\prime} \cdot B=b^{\prime}$. Let $R$ be a region (= connected open subset of $C)$ containing $y$ but having no point or boundary point in $a^{\prime} b^{\prime}$ $+A$. Then $R$ contains a point $x$ of $S$, and there exist arcs $a b$ and $p x$ satisfying (1). Since $y$ does not belong to $S$, it follows at once that $a b \cdot R \neq 0$. The arcs $a b$ and $p x$ contain arcs $a w$ and $p r$ respectively such that $a w \cdot \bar{R}=w$ and $p r \cdot \bar{R}=r$. Let $H=A+a w+p r$.

* A point set is degenerate or non-degenerate according as it does or does not reduce to a single point. This terminology is due to R. L. Moore. 
Then $a^{\prime} b^{\prime}$ contains an arc $u v$ such that $u v \cdot H=u$ and $u v \cdot B=v$. Let $T$ denote one of the sets aw and $p r$ which does not contain $u$ and let $Z$ denote the other one of these sets. Let $Q$ be a region containing the point $T \cdot \bar{R}$ and containing no point of $Z+u v$. Then $Z+u v$ contains an arc $m n$ and $T+Q+R$ contains an arc $q y$ such that $m n \cdot A \supset m, m n, B \supset m, q y \cdot A \supset q$, and $m n \cdot q y=0$. But this is impossible since $y$ does not belong to $S$. Therefore $S=C$. Accordingly $S$ contains a point $x$ of $B$, and thus there exist two mutually exclusive arcs $a b$ and $p x$ joining $A$ and $B$.

LeMma 2. Every point $x$ of $C$ is an interior point of some arc axb in $C$.

This is obvious if $x$ is a cut point of some region $R$ in $C$; for then it is only necessary to take $a$ and $b$ in different components of $R-x$ and any arc $a b$ in $R$ will contain $x$. Thus we may suppose that $x$ is a cut point of no region in $C$. Now let $a$ and $b$ be any two distinct points of $C-x$. Let $R_{1}$ be a region containing $x$ of diameter $<1$ so that $\bar{R}_{1} \cdot(a+b)=0$. There exists a locally connected subcontinuum $E_{1}$ of $C$ of diameter $<1$ which contains $\bar{R}_{1}$ but does not contain either $a$ or $b$. Since $x$ is not a cut point of $R_{1}$, it cannot be a cut point of $E_{1}$; and since it is not an end point of $E_{1}$ it therefore (see appendix below) lies in some non-degenerate cyclic element $C_{1}$ of $E_{1}$. By Lemma 1 there exist in $C$ two mutually exclusive arcs $a a_{1}$ and $b b_{1}$ where $a a_{1} \cdot C_{1}=a_{1}$ and $b b_{1} \cdot C_{1}=b_{1}$. Obviously, we may suppose $a_{1} \neq x \neq b_{1}$. Let $R_{2}$ be a region in $C_{1}$ containing $x$ of diameter less than $1 / 2$, such that $\bar{R}_{2} \cdot\left(a_{1}+b_{1}\right)=0$. There exists a locally connected subcontinuum $E_{2}$ of $C_{1}$ of diameter less than $1 / 2$ which contains $\bar{R}_{2}$ but does not contain either $a_{1}$ or $b_{1}$. Again we may suppose that $x$ cuts no region in $C_{1}$; and it follows just as in the case of $E_{1}$ that $x$ lies in some non-degenerate cyclic element $C_{2}$ of $E_{2}$. By Lemma 1 there exist in $C_{1}$ two mutually exclusive arcs $a_{1} a_{2}$ and $b_{1} b_{2}$ such that $a_{1} a_{2} \cdot C_{2}=a_{2}$ and $b_{1} b_{2} \cdot C_{2}=b_{2}$. Let $R_{3}$ be a region in $C_{2}$ of diameter less than $1 / 3$, and so on. If we continue this process indefinitely, it is clear that the point set

$$
x+a a_{1}+a_{1} a_{2}+a_{2} a_{3}+\cdots+b b_{1}+b_{1} b_{2}+\cdots
$$

so obtained is an arc $a x b$ in $C$.

THEOREM. $C$ is cyclicly connected.

Let $a$ and $b$ be any two points of $C$. Now every point $x$ of $C$ 
lies on some simple closed curve in $C$; for by Lemma 2 there exists an arc $p x q$ in $C$ and there exists an arc $p y q$ in $C-x$, and clearly $p x q+p y q$ contains a simple closed curve containing $x$. Thus there exist simple closed curves $C_{a}$ and $C_{b}$ in $C$ containing $a$ and $b$, respectively. Now if $C_{a} \cdot C_{b}=0$, then by Lemma 1 there exist mutually exclusive arcs $m n$ and $u v$ in $C$ where $m n \cdot C_{a}=m$, $m n \cdot C_{b}=n, u v \cdot C_{a}=u$, and $u v \cdot C_{b}=v$; and in this case clearly the set $m n+\operatorname{arc} n b v$ of $C_{b}+u v+\operatorname{arc} u a m$ of $C_{a}$ is a simple closed curve in $C$ containing $a+b$. If $C_{a} \cdot C_{b}=p$, a single point, then $C-p$ contains an arc $u v$ so that $u v \cdot C_{a}=u$ and $u v \cdot C_{b}=v$; and in this case $u v+\operatorname{arc} v b p$ of $C_{b}+\operatorname{arc} p a u$ of $C_{a}$ is a simple closed curve in $C$ containing $a+b$. Finally, if $C_{a} \cdot C_{b}$ contains more than one point, then $C_{b}$ contains an $\operatorname{arc} p b q$, where $p b q \cdot C_{a}=p+q$; and in this case $p b q+\operatorname{arc} p a q$ of $C_{a}$ is a simple closed curve in $C$ containing $a+b$. Thus the cyclic connectivity theorem is established.

3. Appendix. For the sake of completeness, proofs will now be given for that part of the cyclic element theory which has been used in the above demonstration of the cyclic connectivity theorem.

Definition. A cyclic element of our space $M$ is either a cut point of the space or a set $M_{p}$, where $p$ is a non-cut point and $M_{p}$ is the set of all points which are not separated from $p$ by any single point. (See references in \$1.)

(1) $M_{p}=p$ only when $p$ is an end point.

For suppose $M_{p}=p$, and let $\epsilon$ be any positive number. Since $p$ is not a cut point of $M$, there exists a $\delta, 0<\delta<\epsilon$, such that $M-V_{\epsilon}(p)$ is a subset of some single component $N$ of $M-V_{\delta}(p)$, where $V_{\epsilon}(p)$ denotes the set of all points whose distance from $p$ is $\langle\epsilon$, and similarly for $\delta$. Let $p q$ be an arc such that $p q \cdot N=q$. There exists a point $x$ which separates $p$ and $q$, because $M_{p}=p$. Clearly $x \subset p q$. Thus $x \cdot N=0$ and, as $N$ is connected, $x$ separates $p$ and $N$. Hence $x \in$-separates $p$, and therefore $p$ is an end point.

(2) Every $M_{p}$ has the property that each component $N$ of $M-M_{p}$ has just one limit point in $M_{p}$.

For if $M_{p}$ contains two limit points of $N$, then since clearly $M_{p}$ is closed, it follows that there exists an arc $a b$ such that $a b \cdot M_{p}=a+b$. Hence if $q$ is a point of $a b-(a+b)$, some point $x$ separates $p$ and $q$. But then $x$ necessarily separates $q$ and the set $M_{p}-x$, which is impossible since we have the subarcs $q a$ and $q b$ of $a b$ joining $q$ and the set $M_{p}$.

(3) If the set $Z$ is connected, so also is $Z \cdot M_{p}$ (when non-vacuous).

If, on the contrary, $Z \cdot M_{p}=H_{1}+H_{2}$, where $H_{1}$ and $H_{2}$ are mutually separated, then let $Z$ be divided into two sets $Z_{1}$ and $Z_{2}$ in such a way that these sets contain $H_{1}$ and $H_{2}$, respectively, and any other point $x$ of $Z$ belongs to $Z_{1}$ or to $Z_{2}$ according as the boundary point of the component of $M-M_{p}$ containing $x$ belongs to $H_{1}$ or to $H_{2}$. Obviously $Z_{1} \cdot Z_{2}=0$. And if a point $x$ of one of these sets, say of $Z_{2}$, is a limit point of the other, $Z_{1}$, then since, by virtue of 
the local connectivity of $M$, any component of $M-M_{p}$ containing points of $Z_{2}$ is a neighborhood of any one of its points and contains no point of $Z_{1}$, it follows that $x$ belongs to $H_{2}$. But then if $V$ is a region containing $x$ but containing no point of $H_{1}, V$ contains a point $y$ of $Z_{1} \cdot\left(M-M_{p}\right)$, and clearly this is impossible because the boundary point of the component of $M-M_{p}$ containing $y$ belongs to $H_{1}$ and hence not to $V$.

(4) Every $M_{p}$ is closed, connected, and locally connected and has no cut point.

Taking $Z=M$, we have $Z \cdot M_{p}=M_{p}$; and thus by (3) it follows that $M_{p}$ is connected. Similarly, since each pair of sufficiently near points of $M_{p}$ lie together in a connected subset of $M$ of arbitrarily small diameter and the product of this connected set by $M_{p}$ is connected, it follows that $M_{p}$ is locally connected. Obviously $M_{p}$ is closed. Finally, if some point $x$ cuts $M_{p}$, let $a$ and $b$ be points lying in different components of $M_{p}-x$. Now $a$ and $b$ must lie together in some component $N$ of $M-x$, for otherwise $x$ would separate either $a$ or $b$ from $p$ in $M$. But by (3), $N \cdot M_{p}$ is connected, which is absurd, because $N$ does not contain $x$. Therefore $M_{p}$ has no cut point.

\section{The Johns Hopkins University}

